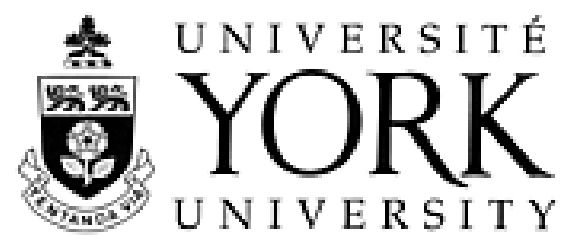

On the Soundness and Completeness of Equational Predicate Logics

George Tourlakis

Technical Report CS-1998-08

November 2, 1998

Department of Computer Science

4700 Keele Street North York, Ontario M3J 1P3 Canada 


\title{
On the soundness and completeness of Equational Predicate Logics
}

\author{
George Tourlakis, York University \\ tgeorge@yorku.ca
}

\begin{abstract}
We present two different formalizations of Equational Predicate Logic, that is, first order logic that uses Leibniz's substitution of "equals for equals" as a primary rule of inference.

We prove that both versions are sound and complete. A by-product of this study is an alternative proof to that contained in [GS3], that the "full" Leibniz rule is strictly stronger than the "no-capture" Leibniz rule, this result obtained here for a complete Logic.

We also show that under some reasonable conditions, propositional Leibniz, no-capture Leibniz, and a full-capture version are all equivalent, provided that the latter is restricted to act on universally valid premises whenever capture is allowed.
\end{abstract}

\section{Introduction.}

Equational Logic, as proposed by Dijkstra and Scholten [DSc], and propounded in the recent text and papers by Gries and Schneider [GS1, GS2, GS3], is finding its way in Computer Science curricula towards satisfying the "Discrete Math" requirements therein.

It is argued by its proponents that Equational Logic is the pedagogically proper setting to do proofs - i.e., work within the theory. It is also a fair observation that Equational Logic may be more cumbersome than "standard" approaches (e.g., [Sh, En, Man, Men]) when it becomes the object of study, in the metatheory - although this phenomenon depends on what semantics we wish to implement. ${ }^{\dagger}$

The department of Computer Science at York University is currently using the equational approach to teaching logic to its first year undergraduates. Understandably, as [GS1] is addressed to undergraduates - and therefore it emphasizes the theory at the expense of the metatheory - it does not address the question of either soundness or completeness of its Equational Predicate Calculus. $\ddagger$ Indeed [GS1] leaves most of the syntax (and semantics) of the first order language where this Calculus is to be "spoken" up to the discretion of the instructor. The set of axioms in [GS1] has a high degree of redundancy, and the pivotal rule, Leibniz, is given under many guises which may be somewhat confusing. Moreover it is very hard to ascertain which rules of inference the authors of [GS1] wanted to be applicable to "universally valid" formulas only, and which ones to be applicable on any premises without restriction. (This uncertainty has triggered many spirited, but productive, e-mail exchanges with

\footnotetext{
$\dagger$ If " $A$ logically implies $B$ " means that every model of $A$ is a model of $B$, then we have a more cumbersome metatheory (e.g., a cumbersome Deduction Theorem, [Sh, Men]). If on the other hand it means "every instantiation of the object variables that makes $A$ true also makes $B$ true", then the metatheory is easier. In particular, a surprisingly easy to prove - and apply-Deduction Theorem becomes available ([Bou, En]).

$\ddagger[$ GS2] cover these questions for the Propositional fragment of the Calculus.
} 
colleagues at York University who, like the author, are teaching "Discrete Math" using the [GS1] text).

We aim in this paper to provide a precise syntax and semantics of a first order language and classical logic, as faithfully as possible to the spirit of the Equational Logic outlined in [GS1], and then to carefully develop the metatheory to the point that soundness can be derived in a self contained manner. We also address (and prove) completeness by showing that the Equational Logic of [GS1] - as it is systematically reincarnated here - is an extension of some standard first order logic (our "standard" yardstick is the approach taken in Shoenfield $[\mathrm{Sh}])$.

As a by-product, we have reduced the very large number of Predicate Logicspecific axiom schemata offered in [GS1] (Chapters 8 and 9) to just two, and have reduced the additional "Leibniz" rule that is proposed in [GS1] - (8.12) in Chapter 8-to a derived rule status (with a correction). Naturally, these reductions will make Equational Calculus even more attractive, as they will reduce the burden placed on memory of both students and instructors - not to mention that it makes the metamathematics much easier.

Formalizing an informal logic is not easy. One has to try to understand what the "intentions" of the informal version were, and implement them faithfully through the syntactic apparatus. Unfortunately, it is in the nature of "informal intentions" to be vague.

In this instance we inferred, partly from the practices in [GS1] and partly from those in [GS3] the following two important points, which shaped our formalization (sections 1 and 2):

(1) Every rule is supposed to be applicable to arbitrary formulas, not just to "universally valid" formulas. We draw this "inference" especially from [GS3] ([GS1] give short shrift to the concept of proofs from a set of "nonlogical" axioms).

Indeed, all the rules of inference in [GS3] are given in the format (for example) "(Equanimity) $\vdash P, \vdash P \equiv Q \longrightarrow \vdash Q$ " where $\longrightarrow$ is metalogical.

In [GS3], since all the rules are given in exactly this format - even the propositional rules - we had to conclude that the "unary" $\vdash$ must mean "deducible from some (unspecified) nonlogical axioms", otherwise this logic would not be usable as a tool for reasoning in any mathematical theory! $!^{\dagger}$

This usage of " $\vdash$-notation" is widespread - e.g., $[\mathrm{Sh}]$ writes " $\vdash A$ " meaning that $A$ is provable from some (unspecified) nonlogical axioms, but on occasion writes $\vdash_{T}$ and $\vdash_{T^{\prime}}$ if deductive power of two sets of nonlogical axioms, $T, T^{\prime}$ is being compared.

(2) Should we, or should we not, restrict the rule "Leibniz" so that no "capture" of object variables occurs during its application?

\footnotetext{
†Obviously, one cannot, e.g., "prove $p \vee q$ from $p$ ", if every rule is undefined except when it applies to universally valid premises. With such rules, all theorems have to be universally valid - and $p \vee q$ is not.
} 
The definitive answer was furnished by the proof-sketch of (9.16) (Metatheorem "generalization") in [GS1]. A pivotal step in the proof is that, once $A \equiv$ true is deduced, then, $(\forall x) A \equiv(\forall x)$ true follows by Leibniz.

Since $A$ is expected to have $x$ free in the "interesting case", clearly, variable capture during an application of Leibniz is allowed in [GS1]. ${ }^{\dagger}$

The layout of the paper is as follows: Section 1 introduces the formal language. Section 2 builds a theory on top of this language (we refer to any such theory as an E-theory or E-logic). Section 3 introduces a host of metatheoremsthat we will use towards the metatheoretical results of sections 4,5 (on soundness and completeness, respectively) and 6 -including the Deduction Theorem. The latter is heavily used in section 6 , where the equivalence between our version of E-logic and the "original" version of [GS1] is proved. Most of the technical apparatus developed in this paper was aimed at making the proofs in section 6 "accessible". The "main lemma" in sections $4-5$ is lemma 5.3 that shows the eliminability of propositional variables. In section 7 we look at an alternative formalization, based on a weaker form of the Leibniz rule that avoids object variable "capture". In the course of the discussion it emerges that "no-capture Leibniz" is equivalent to a "propositional" version of Leibniz and to a full capture version - which restricts its premises to be provable with no nonlogical axioms in those cases that capture occurs. It is also seen that either of these formulations is strictly weaker than "full" Leibniz (Inf2 of section 2) providing a simple alternative proof to a result of [GS3].

A word on the proof of the Deduction Theorem is pertinent here. We opted to do it the "hard way", that is, do it within the Equational Calculus setting rather than in an equivalent "standard" calculus. ${ }^{\ddagger}$ Handling the "Leibniz" rule in the present setting required a few lemmata and a second induction (on formulas) within the proof, making it uncharacteristically long (compare with the proof in [Sh]). This anecdote fortifies our belief that the Equational Calculus is best suited to do proofs, rather than study them.

\section{Syntax}

We think of a first order language, $L$, as triple $L=(V$, Term, Wff $)$, where $V$ is the alphabet, i.e., the set of basic syntactic objects (symbols) that we use to built "terms" and "formulas". We start with a description of $V$, and then we describe the set of terms (Term) and the set of formulas (Wff).

\section{Alphabet}

\footnotetext{
†One cannot gloss over this by "dummy renaming". Interestingly, in [GS3] it is stated that the Leibniz in [GS1] avoids capture. This must be a typo in [GS3], given the "evidence" to the contrary.

¥This would necessitate a metaproof of that equivalence, and in view of the presence of propositional variables and the rule of substitution in [GS1] — none of which are present in, say, [Sh, En, Men, Man, Ba] — would lengthen our exposition in a different, unnecessary, direction.
} 
1. Object variables. An enumerable set $x_{1}, x_{2}, \ldots$ We normally use the metasymbols $x, y, z, u, v, w$ with or without primes to stand for (object) variables.

2. Boolean (or Sentential, or Propositional) variables. An enumerable set $v_{1}, v_{2}, \ldots$ We normally use the metasymbols $p, q, r$ with or without primes to stand for Boolean variables.

(2) These variables will be place-holders for well-formed formulas. This is a II necessary contraption to allow the Substitution rule of inference, to simulate axiom schemata, since the latter are not allowed in the "propositional part" of [GS1]..$^{\dagger}$

3. Equality (between "objects" - see below for its syntactic role), "=".

4. Brackets, ( and ).

5. The Boolean or propositional connectives, $\equiv, \Rightarrow, \vee, \wedge, \neg$.

6. The quantifier, $\forall$ (the existential quantifier, $\exists$, will be taken to be a metasymbol, introduced definitionally).

7. The special Boolean (propositional) constants true and false.

8. A set of symbols (possibly empty) for constants. We normally use the metasymbols $a, b, c, d, e$, with or without subscripts, to stand for constants unless we have in mind some alternative "standard" notation in selected areas of application of the 1st order logic (e.g., $\emptyset, 0, \omega$, etc.).

9. A set of symbols for predicates or relations (possibly empty) for each possible "arity" $n>0$. We normally use $P, Q, R$ with or without primes to stand for predicate symbols.

10. Finally, a set of symbols for functions (possibly empty) for each possible "arity" $n>0$. We normally use $f, g, h$ with or without primes to stand for function symbols.

1.1 Remark. Any two symbols mentioned in items 1-10 are distinct. Moreover (if they are built from simpler "sub-symbols", e.g., $x_{1}, x_{2}, x_{3}, \ldots$ might really be $x|x, x\|x, x \mid\| x, \ldots$ ), none is a substring (or subexpression) of any other.

\section{Terms and Formulas}

\footnotetext{
${ }^{\dagger}$ In [GS3], while substitution is dropped in favour of adopting axiom schemata throughout, propositional variables are retained, presumably to facilitate statement and application of the Leibniz rule.
} 
1.2 Definition. (Terms) The set of terms, Term, is the $\subseteq$-smallest set of strings or "expressions" over the alphabet 1-10 with the following two properties:

Any of the items in 1 or $8(a, b, c, x, y, z$, etc.) are in Term.

If $f$ is a function ${ }^{\ddagger}$ of arity $n$ and $t_{1}, t_{2}, \ldots, t_{n}$ are in Term, then so is the string $f t_{1} t_{2} \ldots t_{n}$.

1.3 Definition. (Atomic Formulas) The set of atomic formulas, Af, contains precisely:

1) The symbols true, false, and every Boolean variable (that is, $p, q, \ldots$ ).

2) The strings $t=s$ for every possible choice of terms $t, s$.

3) The strings $P t_{1} t_{2} \ldots t_{n}$ for every possible choices of $n$-ary predicates $P$ (for all choices of $n>0$ ) and all possible choices of terms $t_{1}, t_{2}, \ldots, t_{n}$.

1.4 Definition. (Well-Formed Formulas) The set of well-formed formulas, Wff, is the $\subseteq$-smallest set of strings or "expressions" over the alphabet 1-10 with the following properties:

\section{a) $\mathbf{A f} \subseteq \mathbf{W f f}$.}

b) If $A, B$ are in $\mathbf{W f f}$, then so are $(A \equiv B),(A \Rightarrow B),(A \wedge B),(A \vee B)$.

c) If $A$ is in Wff, then so is $(\neg A)$.

d) If $A$ is in Wff and $x$ is any object variable (which may or may not occur (as $a$ substring) in the formula $A)$, then the string $((\forall x) A)$ is also in $\mathbf{W f f}$.

We say that $A$ is the scope of $(\forall x)$.

1.5 Remark. (1) $A, B$ in the definition are so-called syntactic or meta-variables, used as names for (arbitrary) formulas. In general, we will let the letters $A, B, C, D, E$ (with or without primes) be names for well-formed formulas, or just formulas as we often say.

(2) We introduce a meta-symbol $(\exists)$ solely in the metalanguage via the definition " $((\exists x) A)$ stands for, or abbreviates, $(\neg((\forall x)(\neg A)))$."

(3) We often write more explicitly, $((\forall x) A[x])$ and $((\exists x) A[x])$ for $((\forall x) A)$ and $((\exists x) A)$. This is intended to draw attention to the variable $x$ of $A$, which

\footnotetext{
‡We will omit the qualification "symbol" from terminology such as "function symbol", "constant symbol", "predicate symbol".
} 
has now become "bound". Of course, notwithstanding the notation $A[x]$ (which only says that $x$ may occur in $A), x$ might actually not be a substring of $A$. In that case, intuitively, $((\forall x) A),((\exists x) A)$ and $A$ "mean" the same thing. This intuition is actually captured by the axioms and rules of inference, as we show in section 3 (3.7).

(4) In [GS1] the preferred metanotation for $((\forall x) A)$ and $((\exists x) A)$ is $(\forall x \mid$ : $A)$ and $(\exists x \mid: A)$, respectively. They also have special (meta) notation for "bounded" quantification, that is $(\forall x \mid B: A)$ and $(\exists x \mid B: A)$ stand for $((\forall x)(B \Rightarrow$ $A))$ and $((\exists x)(B \wedge A))$ respectively. In [GS1] the " $B$ " part is called the "range" of the quantification.

(5) To minimize the use of brackets in the metanotation we adopt standard priorities, that is, $\forall, \exists$, and $\neg$ have the highest, and then we have (in decreasing order of priority) $\wedge, \vee, \Rightarrow, \equiv$. All associativities are right (this is in variance with [GS1], but is just another acceptable - and common - convention of how to be sloppy in the metalanguage, and get away with it).

(6) The language just defined, $\mathbf{W f f}$, is one-sorted, that is, it has a single sort or type of object variable. If one wants to admit several sorts, e.g., $i_{1}, i_{2}, \ldots$ for variables intended to take integer values upon interpretation, $r_{1}, r_{2}, \ldots$ intended to take real values, $b_{1}, b_{2}, \ldots$ intended to take Boolean values (true or false), etc., then we need a more elaborate definition of syntax.

We will need a different quantifier symbol for each sort, say, $\forall_{i}, \forall_{r}, \forall_{b}$, etc., and for each sort a dedicated "equality" predicate $\left(=_{i},=_{r},=_{b}\right.$, etc.). Moreover, function symbols will have a sort or type associated with them (which will be the type of their right field in the intended interpretation). This will induce sorts (types) to all terms.

In the end of the day, we will turn around and use metanotation, suppressing the sort-subscripts (exactly as [GS1] do) and will let the context fend for the various (intended) sorts in the applications.

For this reason we do not burden the formal definition with many sorts. This deliberate omission will not restrict the applicability (to the multi-sorted case) of the few proof-theoretic and model-theoretic results included here.

It should be stated at once that should we feel the need to employ a Boolean object variable type explicitly, $b_{1}, b_{2}, \ldots$, we would not allow the $b_{i}$ to be the same as the $v_{1}, v_{2}, \ldots$ The former are terms, the latter are (atomic) well formed formulas.

\section{Axioms and Rules of Inference}

The axioms and rules of inference will be chosen from [GS1]. We will not allow schemata in the so-called propositional (or Boolean) axioms, in order to agree with the present [GS1] edition. This decision will necessitate the presence of a substitution rule among the rules of inference. We will deviate somewhat in the choice of axioms outside the propositional domain, to maximize convenience. In particular, we will only adopt one among the proposed axioms in [GS1] (Ax2 
below), and one easy to memorize theorem that occurs in [GS1] (namely, the "standard" axiom-schema of instantiation or specialization) as our axiom Ax3. This apparatus will be sufficient to ensure completeness as we will show.

2.1 Definition. (Axioms and Axiom schemata)

Ax1. Include all propositional calculus axioms from [GS1] (there is no harm to actually include all tautologies, as, e.g., Enderton [En] and Manin [Man] do). ${ }^{\dagger}$

Ax2. (Schema) For every formulas $A$ and $B$ such that the variable $x$ does not occur free in $A$, add

$$
A \vee(\forall x) B \equiv(\forall x)(A \vee B)
$$

NB. The above is (9.5) in [GS1]. It is not necessary to introduce the "range" part.

Ax3. (Schema) For every formula $A$, add

$$
(\forall x) A \Rightarrow A[x:=t], \text { for any term } t
$$

with a condition on $t$. The condition is that during substitution no variable of $t$ (all such are free, of course) was "captured" by a quantifier. We say that " $t$ must be substitutable in $x$ ".

NB. We often see the above written as

$$
(\forall x) A[x] \Rightarrow A[t]
$$

or even

$$
(\forall x) A \Rightarrow A[t]
$$

where the presence of $A[x]$ (or $(\forall x) A$, or $(\exists x) A$ ) and $A[t]$ in the same context means that $t$ replaces all $x$ occurrences in $A$.

Ax4. (Schema) For each object variable $x$, add $x=x$.

Ax5. (Leibniz's characterization of equality-1st order version. Schema) For any formula $A$, any object variable $x$ and any term $t$ that is substitutable in $x$ (in $A$ ), add

$$
x=t \Rightarrow A \equiv A[x:=t]
$$

NB. The above is written usually as

$$
x=t \Rightarrow A[x] \equiv A[t]
$$

or even

$$
x=t \Rightarrow A \equiv A[t]
$$

\footnotetext{
${ }^{\dagger}$ Every propositional calculus formula is in $\mathbf{W f f}$, of course, by definitions $1.2-1.4$.
} 
2.2 Remark. (1) In any formal setting that introduces many-sorts explicitly in the syntax, one will need as many versions of $\mathbf{A x 2}-\mathbf{A x 5}$ as there are sorts.

An alternative formal approach is to have only one sort of object variable but introduce one-place predicates $D_{i}, D_{r}, D_{b}$, etc., in the alphabet-one for each sort - with the intended meanings: $D_{i}(x)$ "says" that $x$ is an integer, $D_{b}(x)$ "says" that $x$ is boolean, etc.

Thus, instead of, say, $\left(\forall_{b} x\right) A$ one would write $(\forall x)_{D_{b}} A$-which is a short form of $(\forall x)\left(D_{b}(x) \Rightarrow A\right)$ - etc.

(2) Axioms Ax4-Ax5 characterize equality between "objects". [GS1], while they employ this type of "Leibniz" in their exposition, they do not seem to actually formally adopt the point of view of Predicate Calculus with equality. In any case, adding these two axioms makes this system (explicitly) applicable to mathematical theories such as number theory and set theory, and does not in any way burden what we would like to outline here. Indeed, we will only use these axioms in section 6.

(3) In $\mathbf{A x} \mathbf{3}$ and $\mathbf{A x} \mathbf{5}$ we imposed the condition that $t$ must to be "substitutable" in $x$. I.e., it should not contain free variables that can be "captured" by quantification, as this would distort and invalidate the formula.

The following example is often given in the literature in order to illustrate what might happen after a mindless substitution:

Take $A$ to stand for $(\exists y) \neg x=y$. Then $(\forall x) A[x] \Rightarrow A[y]$ is

$$
(\forall x)(\exists y) \neg x=y \Rightarrow(\exists y) \neg y=y
$$

and $x=y \Rightarrow A \equiv A[y]$ is

$$
x=y \Rightarrow(\exists y) \neg x=y \equiv(\exists y) \neg y=y
$$

neither of which, obviously, is universally valid.

The meta-remedy (advocated by many, for example, Shoenfield [Sh]) is to move the quantified variable(s) out of harm's way, i.e., rename them so that no quantified variable in $A$ has the same name as any (free, of course) variable in $t$.

This renaming is formally correct (i.e., it does not change the meaning of the formula) as we will see in the "variant" (meta)theorem in section 3. Of course, it is always possible to effect this renaming since we have countably many variables, and only finitely many appear free in $t$ and $A$.

This trivial remedy allows us to render the conditions in $\mathbf{A x} \mathbf{3}$ and $\mathbf{A x 5}$ harmless. Essentially, a $t$ is always "substitutable" after renaming.

2.3 Definition. (Rules of Inference) The following four are the rules of inference. These rules are relations on the set $\mathbf{W} f f$ and are written traditionally as "fractions". We call the "numerator" the premise(s) and the "denominator" the conclusion. 
We say that a rule of inference is applied to the formula(s) in the numerator, and that it yields (or results in) the formula in the denominator.

Any set $S \subseteq \mathbf{W f f}$ is closed under some rule of inference iff whenever the rule is applied to formulas in $S$, it also yields formulas in $S$.

Inf1. (Substitution) For any formulas $A, B$ and any propositional variable $p$ that may or may not occur in $A$ (as a substring)

$$
\frac{A}{A[p:=B]}
$$

with a condition. Namely, if $p$ is in the scope of a quantifier $(\forall x)$, then $x$ must $n o t$ be free in $B$.

Inf2. (Leibniz for formulas) For any formulas $A, B, C$ and any propositional variable $p$ (which may or may not occur in $C$ )

$$
\frac{A \equiv B}{C[p:=A] \equiv C[p:=B]}
$$

Inf3. (Equanimity) For any formulas $A, B$

$$
\frac{A, A \equiv B}{B}
$$

Inf4. (Transitivity of $\equiv$ ) For any formulas $A, B, C$

$$
\frac{A \equiv B, B \equiv C}{A \equiv C}
$$

2.4 Remark. (1) Let $P$ be any 1-ary ("unary") predicate and $a$ any constant. Clearly, $(\forall x)(P x \Rightarrow P x) \Rightarrow P a \Rightarrow P x$ is not universally valid, as an interpretation over $\mathbb{N}$ with $P x$ translated to say " $x$ is even" and $a$ to be "the" 0 shows. Indeed a falsifying instance would be $(\forall x)\left(P^{\mathbb{N}} x \Rightarrow P^{\mathbb{N}} x\right) \Rightarrow P^{\mathbb{N}} 0 \Rightarrow P^{\mathbb{N}} 1$, where $P^{\mathbb{N}}$ denotes the "concrete" interpretation of $P$ over $\mathbb{N}$ that we have just suggested.

Yet, $(\forall x)(P x \Rightarrow p) \Rightarrow P a \Rightarrow p$ is universally valid. This explains the "condition" for the substitution rule.

(2) Leibniz for formulas is the primary rule in [GS1] that supports "equational reasoning". In standard approaches to logic it is not a primary rule, rather it appears as the well known "derived rule" (metatheorem) that if $\Gamma \vdash A \equiv B^{\dagger}$ and if we replace one or more occurrences of the subformula $A$ of a formula $D$ (here $D$ is $C[p:=A]$ ) by $B$, to obtain $D^{\prime}$ (that is $C[p:=B]$ ), then $\Gamma \vdash D \equiv D^{\prime}$. Shoenfield $[\mathrm{Sh}]$ calls this derived rule "the equivalence theorem".

(3) [GS1] use "=" for " $\equiv "$ in contexts where they want the symbol to act conjunctionally, rather than associatively, e.g., in successive steps of an equationalstyle proof.

\footnotetext{
$\dagger$ The meaning of the symbol $\vdash$ is defined in 2.5 .
} 
We next define $\Gamma$-theorems, that is, formulas we can prove from the set of formulas $\Gamma$ (this may be empty).

2.5 Definition. ( $\Gamma$-theorems) The set of $\Gamma$-theorems, $\mathbf{T h m}_{\Gamma}$, is the $\subseteq$-smallest subset of Wff that satisfies the following:

Th1. $\mathbf{T h m}_{\Gamma}$ contains as a subset the closure under $\mathbf{I n f 1} \mathbf{1}^{\dagger}$ of: All the axioms in

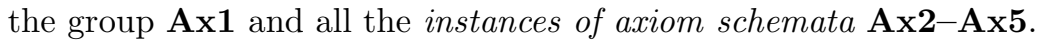

(2) We call the members of this closure the logical axioms.

Th2. $\Gamma \subseteq \mathbf{T h m}_{\Gamma}$. We call every member of $\Gamma$ a nonlogical axiom.

Th3. $\mathbf{T h m}_{\Gamma}$ is closed under each rule Inf2-Inf4.

The (meta)statement $A \in \mathbf{T h m}_{\Gamma}$ is traditionally written as $\Gamma \vdash A$, and we say that $A$ is proved from $\Gamma$ or that it is a $\Gamma$-theorem.

If $\Gamma=\emptyset$, then rather than $\emptyset \vdash A$ we write $\vdash A$. We often say in this case that $A$ is absolutely provable (or provable with no nonlogical axioms).

2.6 Remark. The restriction in the application of Inf1 means that we use it simply to eliminate propositional variables, in particular to turn the axioms of group Ax1 to axiom-schemata. We also, in effect, eliminate its anachronistic presence from our logic, by "applying it up in front" in the course of a deduction. See also 3.24 .

2.7 Definition. ( $\Gamma$-proofs) A finite sequence $A_{1}, \ldots, A_{n}$ of members of $\mathbf{W} \mathrm{ff}$ is a $\Gamma$-proof iff every $A_{i}$, for $i=1, \ldots, n$ is one of

Pr1. A logical axiom (as in Th1 above).

Pr2. A member of $\Gamma$.

Pr3. The result of a rule Inf2-Inf4 applied to (an) appropriate formula(s) $A_{j}$ with $j<i$.

\footnotetext{
$\dagger$ "The closure of a set $\mathcal{S}$ under an operation $\mathcal{O}$ " is the $\subseteq$-smallest set containing the members of $\mathcal{S}$ and closed under $\mathcal{O}$.
} 
2.8 Remark. (1) It is a well known result on inductive definitions that $\Gamma \vdash$ $A$ is equivalent to " $A$ appears in some $\Gamma$-proof" - in the sense of the above definition - and also equivalent to " $A$ is at the end of some $\Gamma$-proof".

(2) It follows from 2.7 that if each of $A_{1}, \ldots, A_{n}$ has a $\Gamma$-proof and $B$ has an $\left\{A_{1}, \ldots, A_{n}\right\}$-proof, then $B$ has a $\Gamma$-proof. Indeed, simply concatenate each of the given $\Gamma$-proofs (in any sequence). Append to the right of that sequence the given $\left\{A_{1}, \ldots, A_{n}\right\}$-proof (that ends with $B$ ). Clearly, the entire sequence is a $\Gamma$-proof, and ends with $B$.

We refer to this phenomenon as the transitivity of $\vdash$.

(3) If $\Gamma \subseteq \Delta$ and $\Gamma \vdash A$, then also $\Delta \vdash A$ as it follows from 2.5 or 2.7. In particular, $\vdash A$ implies $\Gamma \vdash A$ for any $\Gamma$.

(4) The inductive definition of theorems (2.5) allows one to prove properties of $\mathbf{T h m}_{\Gamma}$ by induction on theorems. The equivalent iterative definition, via the concept of proof, allows us a different kind of induction, on the length of proofs.

\section{Some Metatheorems (and the occasional the- orem)}

3.1 Metatheorem. For any formula $A$ and any set of formulas $\Gamma, \Gamma \vdash A$ iff $\Gamma \vdash A \equiv$ true.

Proof. $(\rightarrow)$ In $[\mathrm{GS} 1]$

$$
p \equiv p \equiv \text { true }
$$

is an axiom (from group $\mathbf{A x 1}$ - we are using liberties granted to us by the symmetry and associativity of $\equiv$ ).

Thus, using Inf1 and $[p:=A]$ on (1), we get

$$
\vdash A \equiv A \equiv \text { true }
$$

that is, $A \equiv A \equiv$ true is a logical axiom. Now, 2.8(3) yields

$$
\Gamma \vdash A \equiv A \equiv \text { true }
$$

The assumption $\Gamma \vdash A$, (3) above, and equanimity, yield

$$
\Gamma \vdash A \equiv \text { true. }
$$

$(\leftarrow)$ From [GS1], $\vdash$ true, and hence, for any $\Gamma, \Gamma \vdash$ true.

Thus, the assumption $\Gamma \vdash A \equiv$ true, and equanimity, yield $\Gamma \vdash A$. 
3.2 Theorem. $\vdash(\forall x)$ true $\equiv$ true.

Proof.

$$
\begin{aligned}
& (\forall x) \text { true } \\
& =\langle\text { "Leibniz-rule" and } \vdash \text { true } \vee \text { true } \equiv \text { true }\rangle \\
& (\forall x)(\text { true } \vee \text { true) } \\
& =\langle\mathbf{A x} \mathbf{2}\rangle \\
& \text { true } \vee(\forall x) \text { true } \\
& =\langle\text { by } \vdash \text { true } \vee A \equiv \text { true }\rangle \\
& \text { true }
\end{aligned}
$$

3.3 Metatheorem. For any $\Gamma$, any formula $A$ and any choice of object variable $x, \Gamma \vdash A$ iff $\Gamma \vdash(\forall x) A$.

Proof.

$(\rightarrow)$ We assume that $\Gamma \vdash A$. Thus,

$$
(\forall x) A
$$

$=\langle$ "Leibniz-rule" and Metatheorem 3.1〉

$$
(\forall x) \text { true }
$$

$=\langle$ Theorem 3.2 $\rangle$

true

$(\leftarrow)$ We assume that $\Gamma \vdash(\forall x) A$. By $\mathbf{A x} \mathbf{3}, \vdash(\forall x) A x \Rightarrow A[x:=x]$, that is, $\vdash(\forall x) A \Rightarrow A$, hence $\Gamma \vdash(\forall x) A \Rightarrow A$.

2) $x$ is substitutable in $x$ always. $A$.

By the hypothesis and modus ponens (a valid derived rule), we obtain $\Gamma \vdash$

3.4 Corollary. ("Strong" Generalization) For any formula $A, A \vdash(\forall x) A$.

Proof. Take $\Gamma=\{A\}$ above.

(2) "Strong" implies that there is a weak generalization rule as well. In approaches II to first order logic that contain only propositional rules of inference (e.g., [Bou], [En], where the only rule is modus ponens ${ }^{\dagger}$ ), metatheorem 3.3 requires the constraint - in the only-if direction - that $x$ is not free in any formula of $\Gamma$ that is used in the deduction of $A$. In particular, $A \vdash(\forall x) A$ does not hold (see also $3.23(2))$.

One then has the weak form " $\vdash A$ iff $\vdash(\forall x) A$ ".

Clearly then, the Leibniz rule does not hold in this approach to first order logic.

\footnotetext{
${ }^{\dagger}$ We have here two non-propositional rules, Inf1 and Inf2.
} 
3.5 Corollary. (Substitution) $A\left[x_{1}, \ldots, x_{n}\right] \vdash A\left[t_{1}, \ldots, t_{n}\right]$, for any terms $t_{1}, \ldots, t_{n}$.

Proof. Of course, the $t_{i}$ must be "substitutable" in the respective variables. One can comfortably be silent about this in view of the variant theorem $(3.10$, below).

We illustrate the proof for $n=2$. What makes it interesting is the requirement to have "simultaneous substitution". To that end we first substitute into $x_{1}$ and $x_{2}$ new variables $z, w$-i.e., not occurring in either $A$ or in the $t_{i}$. The proof is the following sequence.

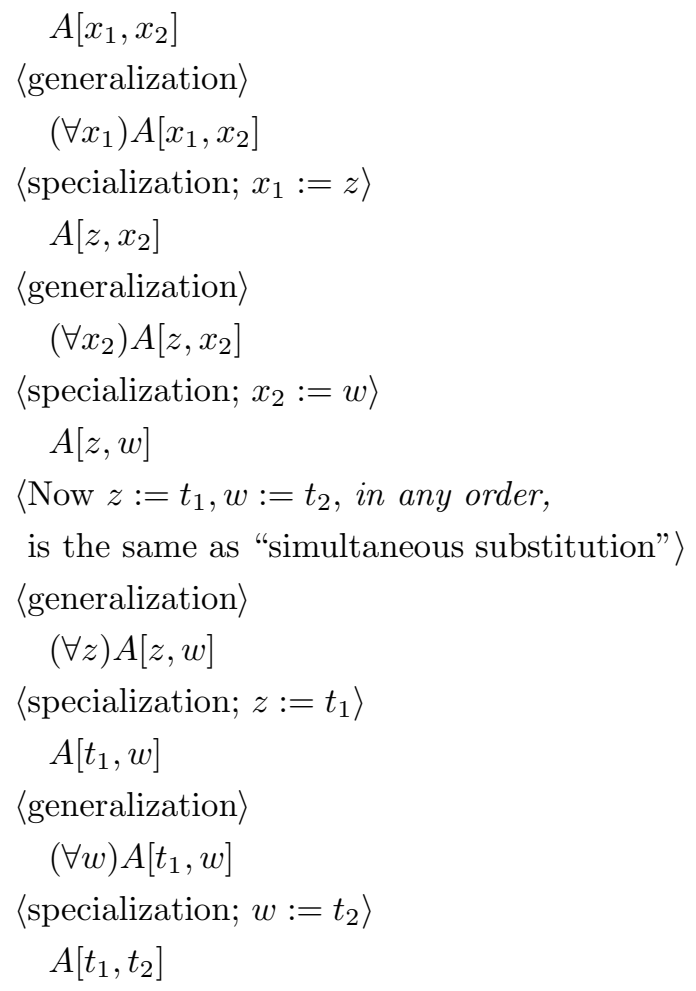

3.6 Theorem. For any formulas $A, B$ such that the object variable $x$ is not free in $A, \vdash A \Rightarrow(\forall x) B \equiv(\forall x)(A \Rightarrow B)$.

Proof. This is so by $\mathbf{A x 2}, \vdash A \Rightarrow B \equiv \neg A \vee B$, and Leibniz for formulas (Inf2).

3.7 Corollary. For any formulas $A$ in which the object variable $x$ is not free, $\vdash A \equiv(\forall x) A$. 
Proof. We have $\vdash(\forall x) A \Rightarrow A$ anyway.

In the equational style we write

$$
\begin{aligned}
& A \Rightarrow(\forall x) A \\
= & \langle\text { by } 3.6\rangle \\
& (\forall x)(A \Rightarrow A) \\
= & \langle\vdash A \Rightarrow A \equiv \text { true } \text { and Leibniz }\rangle \\
= & (\forall x) \text { true } \\
= & \langle\text { by } 3.2\rangle \\
& \text { true }
\end{aligned}
$$

(2) The above Corollary fulfils the promise included in remark 1.5(3). The case for II the metasymbol $\exists$ follows trivially by a sequence of equivalences:

$$
\begin{aligned}
& (\exists x) A \\
& \langle\text { formalizing the "text" }(\exists x) A\rangle \\
& \neg(\forall x) \neg A \\
=\quad & \langle\text { by } 3.7 \text { and Leibniz applied to } \neg p\rangle \\
& \neg(\neg A) \\
= & A
\end{aligned}
$$

In particular, $\vdash(\forall x)$ false $\equiv$ false and $\vdash(\exists x)$ false $\equiv$ false

3.8 Metatheorem. For any formulas $A, B$ such that the object variable $x$ is not free in $A, \Gamma \vdash A \Rightarrow B$ iff $\Gamma \vdash A \Rightarrow(\forall x) B$.

Proof. $(\rightarrow)$ Assume $\Gamma \vdash A \Rightarrow B$.

Then $\Gamma \vdash(\forall x)(A \Rightarrow B)$ by 3.3 , hence $\Gamma \vdash A \Rightarrow(\forall x) B$ by 3.6 (and equanimity).

$(\leftarrow)$ Assume $\Gamma \vdash A \Rightarrow(\forall x) B$.

Then $\Gamma \vdash(\forall x)(A \Rightarrow B)$ by 3.6 (and equanimity), hence $\Gamma \vdash A \Rightarrow B$ by 3.3.

3.9 Corollary. For any formulas $A, B$ such that the object variable $x$ is not free in $A, \Gamma \vdash B \Rightarrow A$ iff $\Gamma \vdash(\exists x) B \Rightarrow A$. 
3.10 Metatheorem. (The variant [or, dummy renaming] metatheorem) For any formula $(\forall x) A$, if $z$ does not occur in it (i.e., is neither free nor bound), then $\vdash(\forall x) A \equiv(\forall z) A[x:=z]$.

NB. We often write this (under the stated conditions) as $\vdash(\forall x) A[x] \equiv(\forall z) A[z]$. Proof. We know that $\vdash(\forall x) A \Rightarrow A[x:=z]$ (Ax3), since $z$ is substitutable in $x$ under the stated conditions. Thus, by 3.3 ,

$$
\begin{gathered}
(\forall z)((\forall x) A \Rightarrow A[x:=z]) \\
=\quad\langle\text { by } 3.6, \text { since } z \text { is not free in }(\forall x) A)\rangle \\
(\forall x) A \Rightarrow(\forall z) A[x:=z]
\end{gathered}
$$

Noting that $x$ is not free in $(\forall z) A[x:=z]$ and is substitutable in $z$ (in $A[x:=z]$ ), we can repeat the above argument to get $\Leftarrow$.

At this point we will be well advised to increase our metatheoretical convenience $e^{\dagger}$ by introducing two important results, namely, Post's tautology Theorem, and (Herbrand's) Deduction Theorem, in that order.

We first offer a careful definition of "tautologies" in Predicate Calculus.

3.11 Definition. (Prime formulas in $\mathbf{W f f}$ ) A formula $A \in \mathbf{W} f f$ is a prime formula iff it is any of

Pri1. Atomic

Pri2. A formula of the form $(\forall x) A$.

Let $\mathcal{P}$ denote the set of all prime formulas in our language. Clearly, $\mathcal{P}$ contains each propositional variable $v_{1}, v_{2}, \ldots$.

That is, a prime formula has no "explicit" propositional connectives (in the case Pri2 any connectives are hidden inside the scope of $(\forall x))$.

Clearly, $A \in \mathbf{W f f}$ iff $A$ is a Propositional Calculus formula over $\mathcal{P}$ (i.e, propositional variables will be all the strings in $\mathcal{P}-\{$ true, false $\})$.

3.12 Definition. (Tautologies in $\mathbf{W f f}$ ) A formula $A \in \mathbf{W f f}$ is a tautology iff it is so when viewed as a Propositional Calculus formula over $\mathcal{P}$.

We call the set of all tautologies, as defined here, Taut. The symbol $\models_{\text {Taut }} A$ says $A \in$ Taut.

The following generalizes 3.12 and will also be needed.

\footnotetext{
${ }^{\dagger}$ The emphasis here is meant to draw a distinction between convenience and "power". Whatever power we have got stems directly from the axioms and the rules of inference, and the definition of $\Gamma$-theorem or $\Gamma$-proof. All else that we add is just "convenience".
} 
3.13 Definition. (Tautologically Implies, for formulas in $\mathbf{W f f}$ ) Given formulas $B \in \mathbf{W}$ ff and $A_{i} \in \mathbf{W}$ ff, for $i=1, \ldots, m$. We say that $A_{1}, \ldots, A_{m}$ tautologically implies $B$, in symbols $A_{1}, \ldots, A_{m} \models$ Taut $B$, iff (viewing the formulas as Propositional Calculus formulas over $\mathcal{P}$ ) every truth assignment to the prime formulas that makes each of the $A_{i}$ true, also makes $B$ true.

(2) While a definition for an infinite set of premises is possible, we will not need it here.

3.14 Metatheorem. (Post's Tautology Theorem) If $A \in \mathbf{W}$ ff is a tautology in the sense of 3.12, then $\vdash A$ with a proof that uses only (substitution instancesunder $\mathbf{I n f 1}$ - of) axioms in the list $\mathbf{A x \mathbf { 1 }}$.

Proof. First, we note the following equivalences. (In the course of this proof, $p, q, r$ stand for any member of $\mathcal{P}-\{$ true, false $\}$, not just for propositional variables of our language.)

$$
\begin{gathered}
\models_{\text {Taut }} \text { true } \equiv \neg p \vee p, \text { and also } \vdash \text { true } \equiv \neg p \vee p \\
\models_{\text {Taut }} \text { false } \equiv \neg p \wedge p, \text { and also } \vdash \text { false } \equiv \neg p \wedge p \\
\models_{\text {Taut }} p \Rightarrow q \equiv \neg p \vee q, \text { and also } \vdash p \Rightarrow q \equiv \neg p \vee q \\
\models_{\text {Taut }} p \wedge q \equiv \neg(\neg p \vee \neg q), \text { and also } \vdash p \wedge q \equiv \neg(\neg p \vee \neg q) \\
\models_{\text {Taut }}(p \equiv q) \equiv((p \Rightarrow q) \wedge(q \Rightarrow p)), \text { and also } \vdash(p \equiv q) \equiv((p \Rightarrow q) \wedge(q \Rightarrow p))
\end{gathered}
$$

where $\vdash$, everywhere above, is achieved by staying within the axiom group $\mathbf{A x 1}$.

Thus, if we transform $A$ into $A^{\prime}$ by applying any sequence of the above equivalences to eliminate all occurrences of true and false and all the connectives except $\neg$ and $\vee$, then we have, on the one hand, that $\models_{\text {Taut }} A^{\prime}$ and on the other hand (by the Leibniz rule) that $\vdash A \equiv A^{\prime}$.

Thus, by equanimity, it suffices to prove $\vdash A^{\prime}$.

A better way to say all this is that, without loss of generality, we assume that the only connectives in $A$ are among $\vee$ and $\neg$ and that the constants true and false do not occur.

Moreover, since $\vdash A \vee A \equiv A$, we may assume without loss of generality that $A$ is a string $A_{1} \vee \cdots \vee A_{n}$ with $n \geq 2$, so that none of the $A_{i}$ is a formula $C \vee D$. We are assuming metanotational abbreviations when it comes to bracketing.

Let us call an $A_{i}$ reducible iff it has the form $\neg(C \vee D)$ or $\neg(\neg C)$. Otherwise it is irreducible. Thus, the only possible irreducible $A_{i}$ have the form $p$ or $\neg p$ (where $p \in \mathcal{P}-\{$ true, false $\}$ ). We say that $A$ is irreducible iff all the $A_{i}$ are.

We define the reducibility degree of $A_{i}$ to be the number of $\neg$ or $\vee$ connectives in it, not counting a possible leftmost $\neg$. The reducibility degree of $A$ is the sum of the reducibility degrees of all its $A_{i}$.

So let $\models_{\text {Taut }} A$, where $A$ is the string $A_{1} \vee \cdots \vee A_{n}, n \geq 2$, where none of the $A_{i}$ is a formula $C \vee D$, and prove (following Shoenfield) by induction on the reducibility degree of $A$ that $\vdash A$. 
For the basis, let $A$ be an irreducible tautology. It must be that $A$ is a string of the form " $\cdots \vee p \vee \cdots \neg p \vee \cdots$ " for some $p$, otherwise (if no $p$ appears both "positively" and "negatively") we can find a truth-assignment that makes $A$ false - a contradiction (assign false to $p$ 's that occur positively only, and true to those that occur negatively only).

Since we have both semantic and syntactic commutativity, let us simplify notation and assume that $A$ is $p \vee \neg p \vee B$. We know enough from [GS1] to conclude that $\vdash p \vee \neg p \vee B$.

Let then $A_{i}$ be reducible. Again, since we have both semantic and syntactic commutativity, let us assume without restricting generality that $i=1$.

We have two cases:

(1) $A$ has the form $\neg(\neg C) \vee D$ (i.e., $A_{1}$ is the string $\neg(\neg C)$ ). Clearly $C \vee D$ is also a tautology, of a lower reducibility degree, hence provable (by Induction Hypothesis - in short, I.H.). Thus,

$$
\begin{array}{cc}
\text { true } \\
=\quad\langle\text { by I.H. }\rangle \\
C \vee D \\
=\quad\langle\text { Leibniz }\rangle \\
\quad \neg(\neg C) \vee D
\end{array}
$$

(2) $A$ has the form $\neg(C \vee D) \vee E$, that is $(C \vee D) \Rightarrow E$ (i.e., $A_{1}$ is the string $\neg(C \vee D)$ ). Clearly $C \Rightarrow E$ and $D \Rightarrow E$ are both tautologies of lower (each) reducibility degree than the original.

By I.H., we have $\vdash C \Rightarrow E$ and $\vdash D \Rightarrow E$. By material in [GS1] ("definition by cases"), we have $\vdash(C \vee D) \Rightarrow E$.

3.15 Corollary. If $A_{1}, \ldots, A_{n} \models_{\text {Taut }} B$, then $A_{1}, \ldots, A_{n} \vdash B$.

Proof. It is an easy semantic exercise to see that

$$
\models_{\text {Taut }} A_{1} \Rightarrow \ldots \Rightarrow A_{n} \Rightarrow B \text {. }
$$

By 3.14

$$
\vdash A_{1} \Rightarrow \ldots \Rightarrow A_{n} \Rightarrow B
$$

hence

$$
A_{1}, \ldots, A_{n} \vdash A_{1} \Rightarrow \ldots \Rightarrow A_{n} \Rightarrow B
$$

Applying modus ponens $n$ times to (1) we get

$$
A_{1}, \ldots, A_{n} \vdash B
$$

2 The above corollary is very convenient. We normally apply it as follows (comII pare with remark 2.8(2)): If each of $A_{1}, \ldots, A_{n}$ has a $\Gamma$-proof (equivalently, is a $\Gamma$-theorem) then so does (equivalently, is) $B$, if $A_{1}, \ldots, A_{n} \models_{\text {Taut }} B$. 
3.16 Corollary. The set of $\Gamma$-theorems, $\mathbf{T h m}_{\Gamma}$, is the $\subseteq$-smallest subset of Wff that satisfies the following:

(i) $\mathbf{T h m}_{\Gamma}$ contains as elements all logical axioms

(ii) $\Gamma \subseteq \mathbf{T h m}_{\Gamma}$

(iii) $\mathbf{T h m}_{\Gamma}$ is closed under any rule

$$
\frac{A_{1}, \ldots, A_{n}}{B}
$$

such that $A_{1}, \ldots, A_{n} \models_{\text {Taut }} B$

(iv) $\mathbf{T h m}_{\Gamma}$ is closed under rule Inf2 (Leibniz).

Proof. NB. This statement is the same as that in definition 2.5, with a difference only in the third clause (compare (iii) here with $\mathbf{T h} 3$ in 2.5).

For a proof, let us call $\widehat{\mathbf{T h m}}_{\Gamma}$ the set inductively defined here, and show that $\widehat{\mathbf{T h m}}_{\Gamma}=\mathbf{T h m}_{\Gamma}$.

$(\subseteq)$ We do induction on $\widehat{\mathbf{T h m}}_{\Gamma}$. We note that $\mathbf{T h m}_{\Gamma}$ satisfies

(1) it contains the same initial objects as $\widehat{\mathbf{T h m}}_{\Gamma}$ (compare Th1-Th2 with $(i)-(i i))$,

(2) it is closed under Inf2,

(3) it is closed under rules (iii), by 3.15 and transitivity of $\vdash$.

The set $\widehat{\mathbf{T h m}}_{\Gamma}$ being the $\subseteq$-smallest with properties (1)-(3), we are done.

() We do induction on $\mathbf{T h m}_{\Gamma}$. We note that $\widehat{\mathbf{T h m}}_{\Gamma}$ satisfies

(4) it contains the same initial objects as $\mathbf{T h m}_{\Gamma}$ (compare $(i)-(i i)$ with Th1-Th2),

(5) it is closed under Inf2,

(6) it is closed under rules Inf3-Inf4 since each of them has its conclusion tautologically implied by the premises(s), so, it is a rule of type (iii).

The set $\mathbf{T h m}_{\Gamma}$ being the $\subseteq$-smallest with properties (4)-(6), we are done once more.

2. Neither of Inf1, Inf2 has the form of a tautological implication.

3.17 Corollary. $\Gamma \vdash A$ iff there is a sequence of formulas $A_{1}, \ldots, A_{n}$ such that $A_{n}$ is $B$ (identical strings), and each $A_{i}$ satisfies one of the following conditions:

(a) $A_{i}$ is a logical axiom,

(b) $A_{i} \in \Gamma$,

(c) For some $A_{j_{m}}$ with $j_{m}<i(m=1, \ldots, r), A_{j_{1}}, \ldots, A_{j_{r}} \models_{\text {Taut }} A_{i}$,

(d) $A_{i}$ is the result of Inf2 on some $A_{j}(<i)$. 
3.18 Lemma. For any formulas $A$ and $B, \vdash(\forall x)(A \Rightarrow B) \Rightarrow(\forall x) A \Rightarrow$ $(\forall x) B$.

Proof. We write an annotated proof (omitting redundant brackets):

$$
(\forall x)(A \Rightarrow B) \Rightarrow A \Rightarrow B
$$

$$
(\forall x) A \Rightarrow A
$$

(3) $\quad(\forall x)(A \Rightarrow B) \Rightarrow(\forall x) A \Rightarrow A$

(4) $\quad(\forall x)(A \Rightarrow B) \Rightarrow(\forall x) A \Rightarrow B$

(5) $\quad(\forall x)((\forall x)(A \Rightarrow B) \Rightarrow(\forall x) A \Rightarrow B)$

(6) $\quad(\forall x)(A \Rightarrow B) \Rightarrow(\forall x) A \Rightarrow(\forall x) B$

$\langle(5), 3.6$, and equanimity $\rangle$

3.19 Lemma. For any $A, B, C$ such that $x$ is not free in $A$,

$$
A \Rightarrow(B \equiv C) \vdash A \Rightarrow((\forall x) B \equiv(\forall x) C) .
$$

Proof. We write an annotated proof:

(1) $\quad A \Rightarrow(B \equiv C)$

(2) $\quad A \Rightarrow(B \Rightarrow C)$

(3) $\quad A \Rightarrow(\forall x)(B \Rightarrow C)$ $\langle x$ not free in $A$. (2) and 3.8 used $\rangle$

$$
(\forall x)(B \Rightarrow C) \Rightarrow(\forall x) B \Rightarrow(\forall x) C
$$

(5) $\quad A \Rightarrow(\forall x) B \Rightarrow(\forall x) C$

A similar proof establishes that $A \Rightarrow(B \equiv C) \vdash A \Rightarrow(\forall x) C \Rightarrow(\forall x) B$.

Since

$$
A \Rightarrow(\forall x) B \Rightarrow(\forall x) C,\left.A \Rightarrow(\forall x) C \Rightarrow(\forall x) B\right|_{\text {Taut }} A \Rightarrow((\forall x) B \equiv(\forall x) C)
$$

we are done by 3.15 .

3.20 Metatheorem. (The Deduction Theorem) If $A$ is closed and $\Gamma, A \vdash B$, then $\Gamma \vdash A \Rightarrow B$.

NB. $\Gamma, A$ means $\Gamma \cup\{A\}$. A converse of the metatheorem is also true, without any restriction on $A$ : That is, $\Gamma \vdash A \Rightarrow B$ implies $\Gamma, A \vdash B$. This follows by modus ponens. We only use the Deduction theorem in section 6 . 
Proof. The proof is by induction on $\Gamma, A$-theorems, as these are characterized in corollary 3.16 .

Basis. Let $B$ be logical. Then, $\vdash B$, and hence $\Gamma \vdash B$.

Trivially, $B \models$ Taut $A \Rightarrow B$, hence, by 3.15 and the transitivity of $\vdash, \Gamma \vdash$ $A \Rightarrow B$.

The same reasoning applies if $B \in \Gamma$, since then $\Gamma \vdash B$ as well.

Finally, if $B$ is the same string as $A$, then $\vdash A \Rightarrow B$, hence $\Gamma \vdash A \Rightarrow B$.

There are only two induction steps, by 3.16 .

1. (Leibniz) Let $\Gamma, A \vdash C \equiv D$, and $B$ be the string $E[p:=C] \equiv E[p:=D]$ for some formula $E$ and propositional variable $p$.

By I.H., $\Gamma \vdash A \Rightarrow(C \equiv D)$.

By induction on the formula $E$, we show that

$$
\Gamma \vdash A \Rightarrow(E[p:=C] \equiv E[p:=D])
$$

Basis. If $E$ is any of true, false, $q, P t_{1} \ldots t_{n}$, or $t_{1}=t_{2}$, then the right hand side of $\Rightarrow$ in (1) reads $E \equiv E$ and the result is trivial (from $\vdash E \equiv E$, and $3.15)$.

If $E$ is $p$, then the right hand side of $\Rightarrow$ in (1) reads $C \equiv D$, and the sought result is identical to the I.H.

Let then $E$ be $\neg G$ (in least parenthesized metanotation). By I.H.,

$$
\Gamma \vdash A \Rightarrow(G[p:=C] \equiv G[p:=D])
$$

thus (1) follows from 3.15 and

$$
A \Rightarrow(G[p:=C] \equiv G[p:=D]) \models \text { Taut } A \Rightarrow(\neg G[p:=C] \equiv \neg G[p:=D]) .
$$

Let next $E$ be $G \circ H$ (in least parenthesized metanotation, where $\circ$ is any of $\equiv, \vee, \wedge, \Rightarrow)$. By I.H.,

$$
\Gamma \vdash A \Rightarrow(G[p:=C] \equiv G[p:=D])
$$

and

$$
\Gamma \vdash A \Rightarrow(H[p:=C] \equiv H[p:=D])
$$

thus (1) follows from 3.15 and

$$
\begin{aligned}
A \Rightarrow(G[p:=C] & \equiv G[p:=D]), A \Rightarrow(H[p:=C] \equiv H[p:=D]) \\
& { } \text { Taut } A \Rightarrow((G \circ H)[p:=C] \equiv(G \circ H)[p:=D])
\end{aligned}
$$

Finally, let $E$ be $(\forall x) G$. By I.H., $\Gamma \vdash A \Rightarrow(G[p:=C] \equiv G[p:=D])$. Since $A$ is closed, $x$ is not free in $A$. Thus, by 3.19 ,

$$
\Gamma \vdash A \Rightarrow(((\forall x) G)[p:=C] \equiv((\forall x) G)[p:=D])
$$

that is, (1) holds. 
2. (Tautological implication rules) Let $\Gamma, A \vdash A_{i}($ for $i=1, \ldots, r)$ and

$$
A_{1}, \ldots, A_{r} \models \text { Taut } B \text {. }
$$

By I.H., $\Gamma \vdash A \Rightarrow A_{i}$ (for $\left.i=1, \ldots, r\right)$. On the other hand, (2) yields ${ }^{\dagger}$

$$
A \Rightarrow A_{1}, \ldots, A \Rightarrow A_{r} \models \text { Taut } A \Rightarrow B
$$

Thus (3.15 and transitivity of $\vdash), \Gamma \vdash A \Rightarrow B$.

For easy applicability of 3.20 we need some additional results.

3.21 Metatheorem. (Metatheorem on constants) Let e be a new constant symbol, added to the alphabet $V$ of the language $L$ resulting to the language $L^{\prime}$. Let $\Gamma^{\prime}$ denote the theory over $L^{\prime}$ that has precisely the same nonlogical axioms as $\Gamma$.

Then, $\Gamma^{\prime} \vdash A[e]$ implies $\Gamma \vdash A[x]$, for any variable $x$ that appears nowhere in the $\Gamma^{\prime}$-proof, as either a free or a bound variable.

Proof. We do induction on $\Gamma^{\prime}$-theorems, using 3.16 .

Basis. If $A[e]$ is logical, then so is $A[x] . A[e]$ cannot be nonlogical in $\Gamma^{\prime}$.

Tautological implication. If $\Gamma^{\prime} \vdash B_{i}[e]$, for $i=1, \ldots, n$, and $B_{1}[e], \ldots$, $B_{n}[e] \models$ Taut $A[e]$, then also $B_{1}[x], \ldots, B_{n}[x] \models$ Taut $A[x]$. By the I.H. (i.e., $\Gamma \vdash B_{i}[x]$, for $\left.i=1, \ldots, n\right)$, we are done in this case.

Leibniz. Let $\Gamma^{\prime} \vdash B[e] \equiv C[e]$ and $A[e]$ be $D[e][p:=B[e]] \equiv D[e][p:=C[e]]$. Clearly, $A[x]$ is $D[x][p:=B[x]] \equiv D[x][p:=C[x]]$, and we are done by I.H., for one last time.

3.22 Corollary. Let $e$ be a new constant symbol, added to the alphabet $V$ of the language $L$ resulting to the language $L^{\prime}$. Let $\Gamma^{\prime}$ denote the theory over $L^{\prime}$ that has precisely the same nonlogical axioms as $\Gamma$.

Then, for any variable $y, \Gamma^{\prime} \vdash A[e]$ iff $\Gamma \vdash A[y]$.

Proof. (if) Trivially, $\Gamma^{\prime} \vdash A[y]$, hence $\Gamma^{\prime} \vdash A[e]$ by 3.5 .

(only-if) Choose a variable $x$ at first with the same restrictions as in 3.21 , to obtain $\Gamma \vdash A[x]$.

Now apply 3.5 (and 3.10 , to avoid substitutability concerns) to get $\Gamma \vdash$ $A[y]$.

3.23 Remark. (1) 3.22 adds flexibility to 3.20 .

$$
\Gamma \vdash(A \Rightarrow B)\left[x_{1}, \ldots, x_{n}\right]
$$

\footnotetext{
${ }^{\dagger}$ Let a truth assignment make the left of $\models_{\text {Taut }}$ true. The "hard case" is when it makes $A$ true at the same time. This forces all the $A_{i}$ to be true, and by (2), $B$ to be true.

${ }^{\ddagger}$ Thus, in particular, $\Gamma^{\prime}$ is "aware" of some additional facts. For example, $\Gamma^{\prime} \vdash e=e$, whereas " $e=e$ " is meaningless in (the language of) $\Gamma$.
} 
where $\left[x_{1}, \ldots, x_{n}\right]$ is the list of all free variables in $A$, is equivalent (by 3.22) to

$$
\Gamma^{\prime} \vdash(A \Rightarrow B)\left[e_{1}, \ldots, e_{n}\right]
$$

where $e_{1}, \ldots, e_{n}$ are "new" constants.

Since $A\left[e_{1}, \ldots, e_{n}\right]$ is closed, by 3.20 , proving

$$
\Gamma^{\prime}, A\left[e_{1}, \ldots, e_{n}\right] \vdash B\left[e_{1}, \ldots, e_{n}\right]
$$

establishes (2), hence (1).

In practice, one does not perform this step explicitly, but ensures that throughout the $\Gamma, A$-proof, whatever free variables were present in $A$ "behave like constants".

(2) In some expositions the Deduction Theorem is not constrained by requiring that $A$ be closed (e.g., [Bou], and more recently [En]).

Which version is right? They both are. If all the rules of inference are "propositional" (e.g., as in [Bou, En], who only employ modus ponens), then the Deduction theorem is unconstrained. If, on the other hand, the rules of inference manipulate object variables via quantification, then one cannot avoid constraining the application of the deduction Theorem, lest one wants to derive (the invalid) $\vdash A \Rightarrow(\forall x) A$ from the valid $A \vdash(\forall x) A$.

How do [Bou, En] cope with this issue? Well, in their approach they do not have $A \vdash(\forall x) A$. They have instead the weaker $\Gamma \vdash A$ iff $\Gamma \vdash(\forall x) A$, with the condition (in the only-if direction) that $x$ not be free in any formula of $\Gamma$ used in the deduction of $A$.

(3) This divergence of approach in choosing rules of inference has some additional repercussions.

One has to be careful in defining the semantic counterpart of $\vdash$, namely, $\models$ (see next section). One wants the two symbols to "track each other" faithfully (Gödel's completeness theorem). ${ }^{\dagger}$

We state a metatheorem that allows some additional flexibility in substitutions. It shows that we can apply substitution (to a known absolute theorem) post facto.

3.24 Metatheorem. (Substitution in absolute theorems) If $\vdash A$, then $\vdash A[p:=$ $W]$ for any formula $W$ and variable $p$.

Proof. Induction on theorems.

Basis. $A$ is a (logical) axiom. Then $A[p:=W]$ is as well (by 2.5), thus $\vdash A[p:=$ $W]$.

There are only two induction steps, by 3.16 .

\footnotetext{
${ }^{\dagger}$ In $[\mathrm{Men}] \models$ is defined inconsistently with $\vdash$.
} 
1. (Leibniz) $A$ is $C[s:=D] \equiv C[s:=E]$ and $\vdash D \equiv E$ (we may view $s$ as a new variable, in particular, different from $p$ ).

By I.H., $\vdash D[p:=W] \equiv E[p:=W]$, hence $\vdash R[s:=D[p:=W]] \equiv R[s:=$ $E[p:=W]]$ by Leibniz, where $R$ is $C[p:=W]$.

2. $A_{1}, \ldots, A_{m} \models$ Taut $A$ and $\vdash A_{i}(i=1, \ldots, m)$. By I.H., $\vdash A_{i}[p:=W]$ $(i=1, \ldots, m)$, but also $A_{1}[p:=W], \ldots, A_{m}[p:=W] \models_{\text {Taut }} A[p:=W]$.

\section{Soundness}

The easiest way to introduce Tarski semantics is to follow Shoenfield.

4.1 Definition. Given a language $L=(V$, Term, Wff $)$, a structure $\mathfrak{M}=$ $(M, \mathcal{I})$ appropriate for $L$ is such that $M \neq \emptyset$ is a set (the "domain") and $\mathcal{I}$ is a mapping that assigns

(1) to each constant $a$ of $V$ a unique member $a^{\mathcal{I}} \in M$

(2) to each function $f$ of $V$ - of arity $n$ - a unique (total) function $f^{\mathcal{I}}: M^{n} \rightarrow M$

(3) to each predicate $P$ of $V$ - of arity $n$ - a unique set $P^{\mathcal{I}} \subseteq M^{n}$

(4) to each propositional variable $p$ of $V$ a unique member $p^{\mathcal{I}}$ of the two element set $\{\mathbf{t}, \mathbf{f}\}$ (we understand $\mathbf{t}$ as "true" and $\mathbf{f}$ as "false")

(5) moreover we set true $^{\mathcal{I}}=\mathbf{t}$ and false $e^{\mathcal{I}}=\mathbf{f}$, where the use of "=" here is metamathematical (equality on $\{\mathbf{t}, \mathbf{f}\}$ ).

We would now like to take a formula $A$, transform each one of its syntactic ingredients - except free variables - $\mathcal{S}$ into its "concrete" counterpart $\mathcal{S}^{\mathcal{I}}$ to finally obtain $A^{\mathcal{I}}$. We could then say that $A$ is valid in $\mathfrak{M}$, and write $\models_{\mathfrak{M}} A$ to mean that $A^{\mathcal{I}}$ is true for all values (from $M$ ) "plugged into" its free variables. This can be done as follows (among other ways).

4.2 Definition. Given $L$ and a structure $\mathfrak{M}=(M, \mathcal{I})$ appropriate for $L$. $L(\mathfrak{M})$ denotes the language obtained from $L$ by adding in $V$ a unique name $\hat{i}$ for each object $i \in M$. This amends both sets Term, Wff into $\operatorname{Term}(\mathfrak{M}), \mathbf{W f f}(\mathfrak{M})$. Members of the latter sets are called $\mathfrak{M}$-terms and $\mathfrak{M}$-formulas respectively.

We extend $\mathcal{I}$ to the new constants: $\hat{i}^{\mathcal{I}}=i$ for all $i \in M$ (where the metamathematical "=" is that on $M$ ).

All we have done here is to allow ourselves to do substitutions like $[x:=i]$ formally. We do instead, $[x:=\hat{i}]$.

One next gives "meaning" to all closed terms in $L(\mathfrak{M})$. 
4.3 Definition. For closed terms $t$ in $\operatorname{Term}(\mathfrak{M})$ we define the symbol $t^{\mathcal{I}} \in M$ inductively:

(1) If $t$ is any of $a$ (original constant) or $\hat{i}$ (imported constant), then $t^{\mathcal{I}}$ has already been defined.

(2) If $t$ is the string $f t_{1} \ldots t_{n}$, where $f$ is $n$-ary, and $t_{1}, \ldots, t_{n}$ are closed $\mathfrak{M}$ terms, we define $t^{\mathcal{I}}$ to be the object (of $\left.M\right) f^{\mathcal{I}}\left(t_{1}^{\mathcal{I}}, \ldots, t_{n}^{\mathcal{I}}\right)$.

Finally, we give meaning to all closed $\mathfrak{M}$-formulas (that is, $\mathfrak{M}$-sentences).

4.4 Definition. For closed formulas $A$ in $\mathbf{W f f}(\mathfrak{M})$ we define the symbol $A^{\mathcal{I}}$ inductively. In all cases, $A^{\mathcal{I}} \in\{\mathbf{t}, \mathbf{f}\}$.

(1) If $A$ is any of $p$ or true or false, then $A^{\mathcal{I}}$ has already been defined.

(2) If $A$ is the string $t=s$, where $t$ and $s$ are closed $\mathfrak{M}$-terms, then $A^{\mathcal{I}}=\mathbf{t}$ iff $t^{\mathcal{I}}=s^{\mathcal{I}}$ (again, the last two occurrences of $=$ refer to equality on $\{\mathbf{t}, \mathbf{f}\}$ and $M$ respectively).

(3) If $A$ is the string $P t_{1} \ldots t_{n}$, where $P$ is an $n$-ary predicate and the $t_{i}$ are closed $\mathfrak{M}$-terms, then $A^{\mathcal{I}}=\mathbf{t}$ iff $\left(t_{1}^{\mathcal{I}}, \ldots, t_{n}^{\mathcal{I}}\right) \in P^{\mathcal{I}}$.

(4) If $A$ is any of $\neg B, B \wedge C, B \vee C, B \Rightarrow C, B \equiv C$, then $A^{\mathcal{I}}$ is determined by the usual truth tables using the values $B^{\mathcal{I}}$ and $C^{\mathcal{I}}$.

(5) If $A$ is $(\forall x) B$, then $A^{\mathcal{I}}=\mathbf{t}$ iff $(B[x:=\hat{i}])^{\mathcal{I}}=\mathbf{t}$ for all $i \in M$.

4.5 Definition. Let $A \in \mathbf{W}$ ff and $\mathfrak{M}$ be a structure as above. An $\mathfrak{M}$-instance of $A$ is a sentence $\bar{A} \in \mathbf{W f f}(\mathfrak{M})$ obtained from $A$ by substituting various constants $\hat{i}, \hat{j}, \ldots$ into all the free variables of $A$.

We say that $A$ is valid in $\mathfrak{M}$, or that $\mathfrak{M}$ is a model of $A$-in symbols $=\mathfrak{M} A$ iff for all possible $\mathfrak{M}$-instances $\bar{A}$ of $A$ we have $\bar{A}^{\mathcal{I}}=\mathbf{t}$.

For any set of formulas $\Gamma$ from $\mathbf{W f f}, \models_{\mathfrak{M}} \Gamma$ denotes the sentence "M is a model of $\Gamma "$, and means that for all $A \in \Gamma, \models \mathfrak{M} A$.

A formula $A$ is universally valid (we often say just valid) iff every structure appropriate for the language is a model of $A$. We then simply write $\models A$.

4.6 Definition. We say that $\Gamma$ logically implies $A$, in symbols $\Gamma \models A$, to mean that every model of $\Gamma$ is also a model of $A$.

(2) This is the correct definition for "logically implies" in those approaches that II allow "strong generalization" (3.4). In particular, this definition allows that $A \models(\forall x) A$ - as we indeed require in order to "match" the syntactic $A \vdash(\forall x) A$. The flip side of this is that $\models A \Rightarrow B$ and $A \models B$ are not equivalent. 
4.7 Definition. (First order theories) Given a first order language $L=(V$, Term, Wff), a (first order) theory $\Gamma$ over $L$ consists of all the formulas in $\Gamma$ taken as nonlogical axioms, along with the logical axioms (see 2.5), and the rules of inference Inf2-Inf4. $\mathbf{T h m}_{\Gamma}$ are the theorems of the theory.

A pure theory is one with $\Gamma=\emptyset$.

4.8 Remark. In the next section we will consider two different ways to "do logic", and therefore will have two kinds of first order theories. One will be as in the definition above. We will call it an "E-theory", to indicate that the logical axioms and especially the rules of inference support equational reasoning. The other will be as developed in [Sh]. We will call such a theory an "S-theory".

4.9 Definition. (Soundness) A pure theory is sound, iff $\vdash A$ implies $\models A$, that is, iff all the theorems of the theory are universally valid.

4.10 Metatheorem. The pure E-theory of definition 4.7 is sound.

Proof. Let $\vdash A$. Pick an arbitrary structure $\mathfrak{M}=(M, \mathcal{I})$ and do induction on $\emptyset$-theorems to show that $\models \mathfrak{M} A$.

Basis. $A$ is a logical axiom (see 2.5). Now the closure of axioms in group Ax1 under Inf1 is a set of tautologies, hence, by 4.4, if $A$ belongs there, $A^{\mathcal{I}}=\mathbf{t}$.

Note that the form of schemata $\mathbf{A x 2}-\mathbf{A x 5}$ is invariant under an application of Inf1.

If $A$ is an instance of $\mathbf{A x 3}$, then a proof that $\bar{A}^{\mathcal{I}}=\mathbf{t}$ for any $\mathfrak{M}$-instance $\bar{A}$ of $A$ is easily accessible in the literature (e.g., [En], [Men]; [Sh] handles the dual axiom $B[x:=t] \Rightarrow(\exists x) B$, where $t$ is substitutable in $x)$.

The literature also takes care of the equality axioms (Ax4 and Ax5). Let us here look into Ax2.

Say $A$ is

$$
B \vee(\forall x) C \equiv(\forall x)(B \vee C)
$$

where $x$ is not free in $B$. Let

$$
\bar{B} \vee(\forall x) \bar{C} \equiv(\forall x)(\bar{B} \vee \bar{C})
$$

be an $\mathfrak{M}$-instance of (1). We need to show that

$$
(\bar{B} \vee(\forall x) \bar{C})^{\mathcal{I}}=\mathbf{f} \text { iff }((\forall x)(\bar{B} \vee \bar{C}))^{\mathcal{I}}=\mathbf{f}
$$

Assume the left side of iff. Then $(\bar{B})^{\mathcal{I}}=\mathbf{f}$ and $((\forall x) \bar{C})^{\mathcal{I}}=\mathbf{f}$. The latter entails $(\bar{C}[x:=\hat{i}])^{\mathcal{I}}=\mathbf{f}$, for some $i \in M$. Since $x$ is not free in $B,(\bar{B})^{\mathcal{I}} \vee(\bar{C}[x:=\hat{i}])^{\mathcal{I} \dagger}$

$\dagger$ The $\vee$ here is the metamathematical truth function on $\{\mathbf{t}, \mathbf{f}\}$. 
is the meaning of an $\mathfrak{M}$-instance of $B \vee C$, and is, of course, $\mathbf{f}$. Thus, the right side of iff is $\mathbf{f}$ (by 4.4).

The other direction is similar.

We next show that if $A$ is the conclusion of a rule, then it is universally valid if the premises are so. By 3.16, we only look at Leibniz.

Leibniz. Let $A$ be $B[p:=C] \equiv B[p:=D]$ and

$$
\models_{\mathfrak{M}} C \equiv D
$$

By induction on the formula $B$, one proves

$$
\models_{\mathfrak{M}} B[p:=C] \equiv B[p:=D]
$$

The interesting induction step is when $B$ is $(\forall x) E$, and $p$ occurs in $E$-but without loss of generality in neither $C$ nor $D$.

Pick an arbitrary $\mathfrak{M}$-instance of $E[p:=C] \equiv E[p:=D]$.

Compare $(\bar{E}[p:=\bar{C}])^{\mathcal{I}}$ with $(\bar{E}[p:=\bar{D}])^{\mathcal{I}}$. Since, by $(4), \bar{C}^{\mathcal{I}}=\bar{D}^{\mathcal{I}}$, I.H. (on formulas) gives

$$
(\bar{E}[p:=\bar{C}])^{\mathcal{I}}=(\bar{E}[p:=\bar{D}])^{\mathcal{I}}
$$

where the "=" above is on $\{\mathbf{t}, \mathbf{f}\}$. By 4.4 , we get

$$
((\forall x)(\bar{E}[p:=\bar{C}]))^{\mathcal{I}}=((\forall x)(\bar{E}[p:=\bar{D}]))^{\mathcal{I}}
$$

and we are done with both inductions.

(2) A by-product of soundness is consistency. A theory $\Gamma$ is consistent iff $\mathbf{T h m}_{\Gamma} \subset$ Wff (proper subset).

Thus, the pure E-theory is consistent, since, by soundness, false is not provable.

\section{Completeness}

5.1 Definition. A theory $\Gamma$ is complete iff $\Gamma \models A$ implies $\Gamma \vdash A$ for any formula A.

One way to show completeness of the pure E-theory is to offer a proof in the style of Henkin. A much easier way is to show that the pure E-theory is at least as powerful as the pure S-theory.

Shoenfield allows no propositional variables, and admits therefore axiom schemata throughout.

The axioms in Shoenfield are

S-Ax1 The schema $A \vee \neg A$

S-Ax2 The dual of Ax3, namely, $A[x:=t] \Rightarrow(\exists x) A$, where $t$ is substitutable in $x$ 
S-Ax3 The E-schemata Ax4 and Ax5.

The rules of inference (after the tautology theorem) boil down to

S-Inf1 Any rule

$$
\frac{A_{1}, \ldots, A_{n}}{B}
$$

is permissible as long as $A_{1}, \ldots, A_{n} \models_{\text {Taut }} B$

\section{S-Inf2}

$$
\frac{A \Rightarrow B}{(\exists x) A \Rightarrow B}
$$

provided $x$ is not free in $B$.

Let us write $\vdash_{\text {theory }} A$ (where "theory" is " $\mathrm{E}$ " or "S") to indicate what axioms and what rules were responsible for the proof of $A$. We have at once,

5.2 Metatheorem. For any $A$ that contains no propositional variables, $\vdash_{S} A$ implies $\vdash_{E} A$.

Proof. The trivial proof on theorems (of S) is omitted. We only note that $\vdash_{\mathrm{E}} A \vee \neg A$ by 3.14 .

In order to prove next that $\models A$ implies $\vdash_{\mathrm{E}} A$, via $\mathrm{S}$, we need a simple technical Lemma.

5.3 Lemma. Let $A$ be a formula over the language $L$ of section 1 , and let $p$ be a propositional variable that occurs in $A$.

Extend the language $L$ by adding $P$, a new 1-ary predicate symbol.

Then, $\models A$ iff $\models A[p:=(\forall x) P x]$ and $\vdash_{E} A$ iff $\vdash_{E} A[p:=(\forall x) P x]$.

Proof. $(\models)$ The only-if is by soundness (substitution 3.24 was used).

For the if-part pick any structure $\mathfrak{M}=(M, \mathcal{I})$ and let $\bar{A}$ be an $\mathfrak{M}$-instance of $A$. Expand $\mathfrak{M}$ to $\mathfrak{M}^{\prime}=\left(V, \mathcal{I}^{\prime}\right)$ where $\mathcal{I}^{\prime}$ is the same as $\mathcal{I}$, except that it also gives meaning to $P$ as follows: If $p^{\mathcal{I}}=\mathbf{t}$, set $P^{\mathcal{I}^{\prime}}=M$, else set $P^{\mathcal{I}^{\prime}}=\emptyset$. Clearly, $p^{\mathcal{I}^{\prime}}=((\forall x) P x)^{\mathcal{I}^{\prime}}$.

By assumption, $\bar{A}[p:=(\forall x) P x]^{\mathcal{I}^{\prime}}=\mathbf{t}$, hence, $\bar{A}^{\mathcal{I}}=\bar{A}^{\mathcal{I}^{\prime}}=\mathbf{t}$ as well.

$(\vdash)$ The only-if is the result of 3.24 .

For the if part, let $\vdash_{\mathrm{E}} A[p:=(\forall x) P x]$. By induction on $\emptyset$-theorems we show that $\vdash_{\mathrm{E}} A$ as well.

Basis. $A[p:=(\forall x) P x]$ is a logical axiom (see 2.5). If it is a substitution (Inf1) instance of a formula in group Ax1, then it is a tautology. But then so is $A$, hence (by 3.14) $\vdash_{\mathrm{E}} A$. $\mathbf{A x 4}$ is not applicable (it cannot contain $(\forall x) P x$ ). It is clear that if $A[p:=(\forall x) P x]$ is an instance of one of the schemata Ax2-Ax3 or $\mathbf{A x 5}$, then replacing all occurrences of $(\forall x) P x$ in $A[p:=(\forall x) P x]$ by the 
propositional variable $p$ results to a formula of the same form, so $A$ is still an axiom, hence $\vdash_{\mathrm{E}} A$.

Leibniz. Let $\vdash_{\mathrm{E}} B \equiv C$ and $A[p:=(\forall x) P x]$ be $D[q:=B] \equiv D[q:=C]$. Let $B^{\prime}, C^{\prime}, D^{\prime}$ be obtained from $B, C, D$ by replacing every occurrence of $(\forall x) P x$ by $p$. $\vdash_{\mathrm{E}} A$.

By I.H., $\vdash_{\mathrm{E}} B^{\prime} \equiv C^{\prime}$, hence (Leibniz) $\vdash_{\mathrm{E}} D^{\prime}\left[q:=B^{\prime}\right] \equiv D^{\prime}\left[q:=C^{\prime}\right]$, i.e.,

$\models$ Taut. Let

$$
B_{1}, \ldots, B_{r} \models_{\text {Taut }} A[p:=(\forall x) P x]
$$

and $\vdash_{\mathrm{E}} B_{i}(i=1, \ldots, r)$. Clearly $(1)$ is only dependent on the configuration of the propositional connectives, hence $B_{1}^{\prime}, \ldots, B_{r}^{\prime} \models_{\text {Taut }} A$, where $B_{i}^{\prime}$ is obtained from $B_{i}$ by replacing all occurrences of $(\forall x) P x$ by $p$.

Hence $\vdash_{\mathrm{E}} A$.

5.4 Corollary. The pure E-theory of definition 4.7 is complete.

Proof. Let $\mid=A$. Let $A^{\prime}$ be obtained from $A$ by replacing each occurrence of true (respectively false) by $p \vee \neg p$ (respectively $p \wedge \neg p$ ) where $p$ is a propositional variable not occurring in $A$. Let $A^{\prime \prime}$ be obtained from $A^{\prime}$ by replacing each propositional variable $p, q, \ldots$ in it by $(\forall x) P x,(\forall x) Q x, \ldots$ respectively, where $P, Q, \ldots$ are new predicate symbols.

Clearly, by $5.3, \models A^{\prime \prime}$. This formula is in the language of S. Thus, by completeness of $\mathrm{S}, \vdash_{\mathrm{S}} A^{\prime \prime}$.

By $5.2, \vdash_{\mathrm{E}} A^{\prime \prime}$, hence $\vdash_{\mathrm{E}} A^{\prime}$, by 5.3 .

Finally, by Leibniz, $\vdash_{\mathrm{E}} A$.

₹ Completeness and soundness were proved for a pure "E-theory" that has a different (much smaller) axiom set than the one given in [GS1]. The next section shows the equivalence of the present pure theory with the one offered in [GS1].

\section{Comparison with the Equational Logic of [GS1]}

We prove that each rule or axiom on quantification that is offered in [GS1], chapters 8 and 9 , is "derived" (hence redundant or dependent) in the E-logic that we have presented here (definitions 2.1, 2.3 and 2.5). We write $\vdash$ meaning $\vdash_{\mathrm{E}}$ throughout.

Nomenclature and numbers given in brackets are those in [GS1].

A.1 "Leibniz (8.12)" The following two rules are meant to supplement the "propositional" version given in [GS1], towards predicate calculus duty ([GS1], p.148).

$$
\frac{A \equiv B}{(\forall x)(C[p:=A] \Rightarrow D) \equiv(\forall x)(C[p:=B] \Rightarrow D)}
$$


and

$$
\frac{D \Rightarrow(A \equiv B)}{(\forall x)(D \Rightarrow C[p:=A]) \equiv(\forall x)(D \Rightarrow C[p:=B])}
$$

The first is a direct application of Inf2, where, without loss of generality, $p$ occurs only in $C$. (If not, apply Inf2 to $C^{\prime} \Rightarrow D$ instead, where $C^{\prime}$ is $C[p:=q]-q$ a propositional variable not in $C \Rightarrow D$. Be sure to use $q:=A$ and $q:=B$ in the application of Inf2).

(1) is not valid, unless we drop " $D \Rightarrow$ " on the premise side (should read just $A \equiv B$ ), in which case it follows from Inf2 exactly like the first of the two (8.12)-rules.

Indeed, take $D$ to be $x=0, C$ to be $(\forall x) p, A$ to be $x=0$ and $B$ to be true. Then,

$$
\models D \Rightarrow(A \equiv B)
$$

that is

$$
\models x=0 \Rightarrow(x=0 \equiv \text { true })
$$

but

$$
\not=(\forall x)(D \Rightarrow C[p:=A]) \equiv(\forall x)(D \Rightarrow C[p:=B])
$$

that is

$$
\forall \forall(\forall x)(x=0 \Rightarrow(\forall x) x=0) \equiv(\forall x)(x=0 \Rightarrow(\forall x) \text { true })
$$

A.2 "Empty range (8.13)". $\vdash(\forall x)($ false $\Rightarrow A) \equiv$ true. It follows from 3.2, $\vdash$ false $\Rightarrow A \equiv$ true, and Inf2.

A.3 "One-point rule (8.14)". Provided that $x$ is not free in $t$, and $t$ is substitutable in $x$ in $A,^{\dagger} \vdash(\forall x)(x=t \Rightarrow A) \equiv A[x:=t]$.

$(\Rightarrow)$ By $\mathbf{A x} \mathbf{3}, \vdash(\forall x)(x=t \Rightarrow A) \Rightarrow t=t \Rightarrow A[t]$. By 3.5 and $\mathbf{A x 4}$, $\vdash t=t$, hence $\vdash(\forall x)(x=t \Rightarrow A) \Rightarrow A[t]$ by 3.15 .

$(\Leftarrow)$ A standard result (from $\mathbf{A x 4}, \mathbf{A x 5}$ ) is that $\vdash x=y \Rightarrow y=x$ (e.g., $[\mathrm{Sh}])$. By $3.5, \vdash x=t \Rightarrow t=x$, thus $(3.15$ and Ax5) $\vdash A[t] \Rightarrow x=t \Rightarrow A$. As $x$ is not free in $A[t], 3.8$ yields $\vdash A[t] \Rightarrow(\forall x)(x=t \Rightarrow A)$.

A.4 "Distributivity (8.15)".

$\vdash(\forall x)(A \Rightarrow B) \wedge(\forall x)(A \Rightarrow C) \equiv(\forall x)(A \Rightarrow B \wedge C)$.

${ }^{\dagger}$ The substitutability condition is necessary, but not mentioned in [GS1] — see 2.2(3). 
$(\Rightarrow)$ Add $(\forall x)(A \Rightarrow B) \wedge(\forall x)(A \Rightarrow C)$ as a (nonlogical) axiom. Then,

1. $(\forall x)(A \Rightarrow B) \wedge(\forall x)(A \Rightarrow C)$

2. $(\forall x)(A \Rightarrow B)$

$\langle 1$. and 3.15$\rangle$

3. $(\forall x)(A \Rightarrow C)$

$\langle 1$. and 3.15$\rangle$

4. $A \Rightarrow B$

$\langle 2$. and specialization $\rangle$

5. $A \Rightarrow C$

$\langle 3$. and specialization $\rangle$

6. $A \Rightarrow B \wedge C$

$\langle 4 ., 5$. and 3.15$\rangle$

7. $(\forall x)(A \Rightarrow B \wedge C)$

$\langle 6$. and 3.4$\rangle$

By the Deduction Theorem, we are done.

$(\Leftarrow)$ With amended "hints", the above proof can be reversed (7.-1.)

A.5 (8.16)-(8.18) in [GS1] boil down to (8.18) if "*" is " $\forall$ ".

"Range split (8.18)". $(\forall x)(A \vee B \Rightarrow C) \equiv(\forall x)(A \Rightarrow C) \wedge(\forall x)(B \Rightarrow C)$.

$(\Rightarrow)$ Add $(\forall x)(A \vee B \Rightarrow C)$. Then,

1. $(\forall x)(A \vee B \Rightarrow C)$

2. $A \vee B \Rightarrow C$

$\langle 1$. and specialization $\rangle$

3. $A \Rightarrow C$

$\langle 2$. and 3.15$\rangle$

4. $B \Rightarrow C$

$\langle 2$. and 3.15$\rangle$

5. $(\forall x)(A \Rightarrow C)$

$\langle 3$. and 3.4$\rangle$

6. $\quad(\forall x)(B \Rightarrow C)$

$\langle 4$. and 3.4$\rangle$

7. $(\forall x)(A \Rightarrow C) \wedge(\forall x)(B \Rightarrow C)$

$\langle 5 ., 6$. and 3.15$\rangle$

By the Deduction Theorem, we are done.

$(\Leftarrow)$ Reverse the above proof.

A.6 "Interchange of dummies (8.19)".

$(\forall x)(A \Rightarrow(\forall y)(B \Rightarrow C)) \equiv(\forall y)(B \Rightarrow(\forall x)(A \Rightarrow C))$, on the condition that $y$ is not free in $A$ and $x$ is not free in $B$.

$(\Rightarrow)$ Add $(\forall x)(A \Rightarrow(\forall y)(B \Rightarrow C))$. Then,

1. $(\forall x)(A \Rightarrow(\forall y)(B \Rightarrow C))$

2. $A \Rightarrow(\forall y)(B \Rightarrow C)$

$\langle 1$. and specialization $\rangle$

3. $A \Rightarrow B \Rightarrow C$

$\langle 2$. and $3.8 ; y$ not free in $A\rangle$

4. $B \Rightarrow A \Rightarrow C$

$\langle 3$. and 3.15$\rangle$

5. $B \Rightarrow(\forall x)(A \Rightarrow C)$

$\langle 4$. and $3.8 ; x$ not free in $B\rangle$

6. $(\forall y)(B \Rightarrow(\forall x)(A \Rightarrow C))$

$\langle 5$. and 3.4$\rangle$ 
By the Deduction Theorem, we are done.

$(\Leftarrow)$ Reverse the above proof.

A.7 "Nesting (8.20)". $(\forall x)(\forall y)(A \wedge B \Rightarrow C) \equiv(\forall x)(A \Rightarrow(\forall y)(B \Rightarrow C))$, on the condition that $y$ is not free in $A$.

$(\Rightarrow)$ Add $(\forall x)(\forall y)(A \wedge B \Rightarrow C)$. Then,

1. $(\forall x)(\forall y)(A \wedge B \Rightarrow C)$

2. $A \wedge B \Rightarrow C$

$\langle 1$. and specialization $\rangle$

3. $A \Rightarrow B \Rightarrow C$

$\langle 2$. and 3.15$\rangle$

4. $A \Rightarrow(\forall y)(B \Rightarrow C)$

$\langle 3$. and $3.8 ; y$ not free in $A\rangle$

5. $(\forall x)(A \Rightarrow(\forall y)(B \Rightarrow C))$

$\langle 4$. and 3.4$\rangle$

By the Deduction Theorem, we are done.

$(\Leftarrow)$ Reverse the above steps.

A.8 "Dummy renaming (8.21)". This is 3.10 .

A.9 "Trading (9.2)". This is an abbreviation in the metalanguage. $(\forall x)_{B}$ is not a "new quantifier". Thus, $\left((\forall x)_{B} A\right)$ abbreviates $((\forall x)(B \Rightarrow A))$.

A.10 "Distributivity of $\vee$ over $\forall(9.5)$ ". Included as Ax2.

This concludes that for every formula $A, \vdash_{G S 1} A$ implies $\vdash_{E} A$, where $\vdash_{G S 1}$ indicates provability in the system outlined in [GS1].

Conversely, the set of axioms in [GS1] - along with the (corrected) "augmented Leibniz" (8.12) - subsume our version of pure E-theory (our Ax3 is a theorem in [GS1]). Thus,

6.1 Metatheorem. For every formula $A, \vdash_{G S 1} A$ iff $\vdash_{E} A$.

6.2 Corollary. The pure first order theory outlined in [GS1] is sound and complete.

\section{Concluding remarks; an alternative formal- ism for Equational Logic}

We have offered a careful formalization of the Equational Predicate Logic outlined in [GS1] and we have proved that this formalization is sound and complete.

We want to briefly investigate here an alternative formalization of Equational Logic, based on a weaker form of the Leibniz rule. We have already observed 
(see the remark following 3.4) that since Inf2 yields "strong generalization", first order Logics such as [Bou, En] do not support it.

We show how such Logics can be recast in the Equational Logic paradigm.

We base our discussion here, for the sake of concreteness, to the version of first order Logic as it is defined in [En]. We start by describing this logic.

We allow propositional variables in formulas only for the purpose of making the statement of "Leibniz", below, easy. The only connectives are $\neg, \Rightarrow, \forall$. The remaining connectives- $\equiv, \vee, \wedge, \exists$-are introduced as abbreviations, and the same holds for the constants (0-ary propositional connectives in reality) true and false (for example, in view of the theorem $\vdash(A \equiv A) \equiv(B \equiv B)$, we abbreviate any occurrence of $A \equiv A$ by true).

There is only one rule of inference, modus ponens.

The axioms (schemata) are all the possible "partial" generalizations ${ }^{\dagger}$ of the following

En1. All tautologies (in the sense of 3.12),

En2. All formulas of the form $(\forall x) A \Rightarrow A[t]$, provided $t$ is substitutable in $x$,

En3. All formulas of the form $(\forall x)(A \Rightarrow B) \Rightarrow(\forall x) A \Rightarrow(\forall x) B$ (see 3.18),

En4. All formulas of the form $A \Rightarrow(\forall x) A$, provided $x$ is not free in $A$ (see 3.7).

En5. Ax4 and Ax5.

In this logic the following weaker version of Inf2, let us call it no-capture Leibniz, is a derived rule:

$$
\frac{A \equiv B}{C[p:=A] \equiv C[p:=B]}
$$

provided neither $A$ nor $B$ contain free variables that are captured by quantifiers during the above substitutions.

For a proof, we note that the following Metatheorem ("restricted generalization") holds in [En]:

$\Gamma \vdash G$ iff $\Gamma \vdash(\forall x) G$, provided no formula in $\Gamma$ has $x$ free. Here then is the proof of no-capture Leibniz, $A \equiv B \vdash C[p:=A] \equiv C[p:=B]$, by induction on the formula $C$.

Basis. $C$ is atomic. If $C$ is the string $p$, then $C[p:=A]$ is $A$ and $C[p:=B]$ is $B$, so our conclusion is our hypothesis.

In all other cases $C[p:=A] \equiv C[p:=B]$ is the tautology $C \equiv C$.

Induction Step(s) (I.S.).

I.S.1. $C$ is $\neg D$. By I.H., $A \equiv B \vdash D[p:=A] \equiv D[p:=B]$.

Since $D[p:=A] \equiv D[p:=B] \models_{\text {Taut }} \neg D[p:=A] \equiv \neg D[p:=B]$, we are done in this case. ${ }^{\dagger}$

\footnotetext{
${ }^{\dagger}$ A generalization $B$ of $A$ is partial iff $B$ is $A$, prefixed with zero or more strings $(\forall x)-x$ may or may not occur in $A$. The well known "universal closure of $A$ " is a special case.

† The tautology theorem holds in [En].
} 
I.S.2. $C$ is $D \Rightarrow G$. By I.H., $A \equiv B \vdash D[p:=A] \equiv D[p:=B]$ and $A \equiv B \vdash$ $G[p:=A] \equiv G[p:=B]$. Since $D[p:=A] \equiv D[p:=B], G[p:=A] \equiv$ $G[p:=B] \models_{\text {Taut }}(D \Rightarrow G)[p:=A] \equiv(D \Rightarrow G)[p:=B]$, we are done once more.

I.S.3. $C$ is $(\forall x) D$. By I.H., $A \equiv B \vdash D[p:=A] \equiv D[p:=B]$, hence (Tautology Theorem) $A \equiv B \vdash D[p:=A] \Rightarrow D[p:=B]$.

By restricted generalization, since $x$ is not free in $A \equiv B, A \equiv B \vdash$ $(\forall x)(D[p:=A] \Rightarrow D[p:=B])$, hence by modus ponens and En3, $A \equiv$ $B \vdash(\forall x) D[p:=A] \Rightarrow(\forall x) D[p:=B]$.

Similarly (from $A \equiv B \vdash D[p:=A] \Leftarrow D[p:=B]$ ), $A \equiv B \vdash(\forall x) D[p:=$ $A] \Leftarrow(\forall x) D[p:=B]$, hence, one more application of the Tautology Theorem gives $A \equiv B \vdash(\forall x) D[p:=A] \equiv(\forall x) D[p:=B]$. This concludes the proof.

Assume now that we want to do Equational Logic, based on no-capture Leibniz.

We adopt En1-En5 above, but our rules - instead of modus ponens - are Inf3, Inf4 and no-capture Leibniz.

Trivially, modus ponens is a derived rule, and thus [En]-logic and this version of Equational Logic are equivalent. In particular, the latter is sound and complete.

This study also demonstrates, via an entirely different avenue than the one taken in [GS3], that "full Leibniz" (Inf2) is strictly more powerful than the "no-capture" version even in a complete logic. The similar result in [GS3] is shown for an incomplete logic, one in which Ax3 is not deducible.

There are many ways to "massage" the rule Leibniz. We wish to cite here two more versions and gauge their relative power.

First, let us restrict the rule on universally valid formulas only, whenever "capture" occurs in its application. Syntactically, this is rule $L 2$ below.

$\Gamma \vdash A \equiv B$ implies $\Gamma \vdash C[p:=A] \equiv C[p:=B]$ provided $\Gamma=\emptyset$ if capture occurs

The same proof we presented above for the no-capture version (in the logic of [En]) can be re-traced with trivial modifications to yield $L 2$ as a derived rule (restricted generalization with $\Gamma=\emptyset$ is used).

Conversely, adopting En1-En5 above, and the rules - instead of modus ponens - Inf3, Inf4 and $L 2$, we can derive modus ponens:
1. $A$
〈given〉
2. $A \Rightarrow B$
$\langle$ given $\rangle$
3. $\quad$ true $\Rightarrow B$
$\langle 1 ., 2 ., 3.1, L 2$ and Inf3 $\rangle$
4. $B$
$\langle 3$. and 3.15$\rangle$

Thus, in the presence of En1-En5, and Inf3, Inf4, $L 2$ and no-capture Leibniz are equivalent, and the system so founded is equivalent to that in [En]. 
Indeed, the proof of modus ponens above makes it clear that all we really need is a propositional Leibniz, $P L$, that is

$$
\frac{A \equiv B}{C[p:=A] \equiv C[p:=B]}, \text { where } p \text { is not in the scope of a quantifier }
$$

In an application of $P L$, we do not require that $\vdash A \equiv B$.

We can summarize this discussion thus:

7.1 Metatheorem. In the presence of the axioms En1-En5 and the rules Inf3, Inf4, all the following versions of the Leibniz rule are equivalent: PL, "no-capture", and L2. Adopting any of them yields a sound and complete first order logic (equivalent to that in [En]).

In this logic, "full" Leibniz (Inf2) is not derivable.

An instructor teaching Equational Logic to first year students will probably be tempted to "interpret" [GS1] logic in this simpler framework - using $P L$ and demonstrating that both $L 2$ and no-capture Leibniz are derived rules.

This alternative version may well prove more palatable in the classroom, if for no other reason, because of the simplicity of its rules of inference and its support for an unconstrained Deduction Theorem.

Acknowledgements. I warmly thank my colleague Steve Watson who carefully read a previous version of the paper and offered valuable advice. In particular he pointed to a problematic use of Inf1 in my original definition 2.5, which I have now fixed. I am also indebted to my colleague Jonathan Ostroff who encouraged me to simplify the formalism. Heeding his request I came up with the simpler formalism ([En]-based) of section 7 .

\section{Bibliography}

[Ba] Barwise, J. "An introduction to first-order logic", in Handbook of Mathematical Logic (J. Barwise, Ed.), 5-46, Amsterdam: North-Holland Publishing Company, 1978.

[Bou] Bourbaki, N. Élémens de Mathématique; Théorie des Ensembles, Ch. 1, Paris: Hermann, 1966.

[DSc] Dijkstra, E. W., and Scholten, C. S. B. Predicate Calculus and Program Semantics, New York: Springer-Verlag, 1990.

[En] Enderton, H. B. A mathematical introduction to logic, New York: Academic Press, 1972.

[GS1] Gries, D. and Schneider, F. B. A Logical Approach to Discrete Math, New York: Springer-Verlag, 1994. 
[GS2] Gries, D. and Schneider, F. B. Equational propositional logic, Information Processing Letters, 53, 145-152, 1995.

[GS3] Gries, D. and Schneider, F. B. Formalizations of substitution of equals for equals, Pre-print, Sept. 1998 (personal communication).

[Man] Manin, Yu. I. A Course in Mathematical Logic, New York: SpringerVerlag, 1977.

[Men] Mendelson, E. Introduction to mathematical logic, 3rd Edition, Monterey, Calif: Wadsworth \& Brooks, 1987.

[Sh] Shoenfield, J. R. Mathematical Logic, Reading, Mass.: Addison-Wesley, 1967. 